Research Paper

\title{
Acid-electrolyzed functional water-induces Interleukin- $1 \alpha$ release from Intracellular Storage Sites in Oral Squamous Cell Carcinoma
}

\author{
Tomoko Takemoto ${ }^{1,2}$, Ryo Kaetsu1,2, Machiko Hanayama ${ }^{3}$, Yuuichi Ishiyama ${ }^{3}$, Masayuki Sadamura ${ }^{3}$, \\ Kensuke Nishio ${ }^{4,5}$, Mariko Tsunoda ${ }^{6,7}$, Masatake Asano ${ }^{6,7}$ and Mitsuru Motoyoshi1,8 \\ 1. Department of Orthodontics, Nihon University School of Dentistry, Tokyo, Japan. \\ 2. Division of Oral Structural and Functional Biology, Nihon University Graduate School of Dentistry, Tokyo, Japan. \\ 3. Division of Applied Oral Sciences, Nihon University Graduate School of Dentistry, Tokyo, Japan. \\ 4. Department of Complete Denture Prosthodontics, Nihon University School of Dentistry, Tokyo, Japan. \\ 5. Division of Advanced Dental Treatment, Dental Research Center, Nihon University School of Dentistry, Tokyo, Japan, 101-8310 \\ 6. Department of Pathology, Nihon University School of Dentistry, Tokyo, Japan. \\ 7. Division of Immunology and Pathobiology, Dental Research Center, Nihon University School of Dentistry, Tokyo, Japan. \\ 8. Division of Clinical Research, Dental Research Center, Nihon University School of Dentistry, Tokyo, Japan. \\ $\triangle$ Corresponding author: Masatake Asano, Department of Pathology, Nihon University School of Dentistry, Kanda Surugadai, Chiyoda-Ku, Tokyo 101-8310, Japan. \\ Tel/FAX: +81-33219-8124/+81-33219-8340, E-mail: asano.masatake@nihon-u.ac.jp.
}

(C) The author(s). This is an open access article distributed under the terms of the Creative Commons Attribution License (https://creativecommons.org/licenses/by/4.0/). See http://ivyspring.com/terms for full terms and conditions.

Received: 2020.10.02; Accepted: 2021.01.21; Published: 2021.02.18

\begin{abstract}
The aim of this study was to examine the acid-electrolyzed functional water (FW)-mediated cytokine release in an oral squamous cell carcinoma-derived cell line (OSCC) following treatment with FW. FW is generated by the electrolysis of a sodium chloride solution and accelerate the burn wound healing. To elucidate the underlying mechanisms, the cytokine/chemokine secretion profile of HSC3 cells was examined using a cytokine array. FW treatment significantly induced interleukin (IL)-l a secretion, which was confirmed by enzyme-linked immunosorbent assay. Subsequently, the HSC3 cells were pre-treated with cycloheximide (CHX) for $1 \mathrm{~h}$ prior to FW stimulation to determine whether the augmented IL-l $\alpha$ secretion was due to enhanced protein synthesis. CHX pre-treatment did not affect IL-1 a secretion suggesting that the secreted IL-1 a might have been derived from intracellular storage sites. The amount of IL- $1 \alpha$ in the cell lysate of the FW-treated HSC3 cells was significantly lower than that of the non-treated cells. Immunofluorescence staining using a polyclonal antibody against full-length IL- $1 \alpha$ revealed a drastic reduction in IL- $1 \alpha$ inside the FW- treated cells. IL- $1 \alpha$ is synthesized in its precursor form (plL-l $\alpha$ ) and cleaved to produce pro-piece and mature IL-l $\alpha$ (pplL-l $\alpha$ and $\mathrm{mlL}-1 \alpha$ ) inside the cells. In the present study, only plL- $1 \alpha$ was detected within the HSC 3 cells in its resting state. However, FW stimulation resulted in the release of the $33 \mathrm{kDa}$ and two other smaller forms (about $19 \mathrm{kDa}$ ) of the protein. These results indicates that FW treatment induces IL-1 $\alpha$ secretion, a typical alarmin, from the intracellular storage in OSCC cells.
\end{abstract}

Key words: acid-electrolyzed functional water, interleukin-1a, oral squamous cell carcinoma-derived cell line, alarmin

\section{Introduction}

Acid-electrolyzed functional water (FW) is generated by electrolyzing low concentrations of saline [1, 2]. Hypochlorous acid is the main component of $\mathrm{FW}$ at a concentration of approximately 10-60 ppm. The bactericidal effects of FW against drug-resistant bacteria and viruses are more potent than those of other chlorine-based disinfectants due to its acidic properties, despite the relatively lower levels of chloride [3]. Moreover, FW was demonstrated to accelerate major burn healing by preventing burn-wound sepsis [4]. Although the underlying mechanisms have not been elucidated, these beneficial effects of FW might be attributed to increased cytokine release. Previously, we demonstrated that the treatment of several cell lines with FW resulted in the release of various factors $[5,6$, 7]. Among them, the release of extracellular matrix metalloproteinase inducer (EMMPRIN) was 
significantly augmented in the human oral squamous cell carcinoma cell line (OSCC) HSC3 [7]. Augmented EMMPRIN secretion was not regulated at the transcriptional level and appeared to be released from intracellular storage sites. Based on these observations, EMMPRIN was proposed as a novel alarmin [7]. Alarmins are molecules that are rapidly released from damaged cells [8] and function as early warning signals to activate the innate and adaptive immune systems.

In the present study, we examined the cytokine array experiment to identify the cytokines secreted in response to FW. The data demonstrated that IL-1a secretion was exclusively augmented by FW. IL-1a is a proinflammatory cytokine synthesized as a precursor (pIL-1a) inside the cells. pIL-1a is processed by calcium-dependent proteinase calpain within the cell to generate pro-piece IL-1a (ppIL-1a, N-terminal half of pIL-1 $\alpha$ ) and mature mIL-1a (C-terminal half of pIL-1a) [9]. Due to the lack of a signal peptide, mIL-1a is secreted via the endoplasmic reticulum (ER)-Golgiindependent pathway [10]. mIL-1a triggers various downstream inflammatory reactions through its cognate receptors. On the other hand, pIL-1a and ppIL-1a preferentially localize in the nucleus due to the nuclear localizing sequence (NLS) and regulate the expression of its target genes by activating transcription factors such as nuclear factor kappa beta (NF-kB) and activator protein -1 (AP-1) [11]. Moreover, pIL-1a is reported to be released from cells undergoing necrosis $[12,13,14]$. Intriguingly, both pIL-1a and mIL-1a were reported to induce IL-6 secretion from the human lung carcinoma cell line, A549 [15]. Taken together, these findings indicate the role of IL-1a as a typical alarmin and its activity has been demonstrated by the fundamental studies [13, $15,16]$.

We demonstrate here that FW induces IL-1a secretion from the intracellular storage sites of OSCC. The molecular forms of secreted IL-1a is composed of two closely related $19 \mathrm{kDa}$ forms in addition to $33 \mathrm{kDa}$ pIL-1a. As only $33 \mathrm{kDa}$ pIL-1a is released from resting cells, FW might enhance the cleavage of pIL-1a inside the cells.

\section{Materials and methods}

\section{Reagents}

FW, containing $20 \% \mathrm{Cl}_{2}$ and $80 \%$ hypochlorous acid $(\mathrm{pH}, 2.2-2.7)$ was obtained using the Oxilyzer (Miura Denshi, Akita, Japan). Cycloheximide (CHX) was purchased from Sigma-Aldrich (Tokyo, Japan). The calpain inhibitor calpeptin was purchased from MedChemExpress (Monmouth Junction, NJ, USA).

\section{Cell culture and FW treatment}

The HSC3 cells were maintained in RPMI1640 medium supplemented with $10 \%$ fetal calf serum (FCS), $50 \mu \mathrm{g} / \mathrm{ml}$ streptomycin, and $50 \mathrm{U} / \mathrm{ml}$ penicillin, in a $5 \% \mathrm{CO}_{2}$ incubator. The cells $\left(1 \times 10^{5} / 24\right.$ well plate) were washed with phosphate-buffered saline (PBS) three times and treated with FW for $30 \mathrm{~s}$. The reaction was stopped by adding an equal volume of the complete medium. After discarding the used medium, fresh medium was added to the cells, which were then allowed to incubate for 30, 60 and $180 \mathrm{~min}$. For the CHX experiment, the cells were pre-treated with CHX ( 1 and $10 \mu \mathrm{M})$ for $1 \mathrm{~h}$. Then, the cells were treated with or without FW, as described, and cultured. Antibody $(\mathrm{Ab})$ inhibition was performed by treating the cells with or without anti- IL-1receptor type 1 (IL-1R1) Ab (100 ng/ml, Abcam) or a control $\mathrm{Ab}$ for $1 \mathrm{~h}$ prior to FW stimulation. For calpain inhibition, the cells were pre-treated with graded amount of calpeptin $(0.1,1,10 \mu \mathrm{M})$ for $1 \mathrm{~h}$ prior to FW treatment. The lactate dehydrogenase (LDH) activity was measured with Lactate Dehydrogenase Activity Assay Kit (Sigma-Aldrich Japan, Tokyo, Japan).

\section{Cytokine array and ELISA}

HSC 3 cells were plated on to a $10-\mathrm{cm}$ cell culture plate at a density of $1 \times 10^{6}$ cells on the day before the experiment. The cells were treated with or without FW for $30 \mathrm{~s}$. After washing, the cells were cultured for another $18 \mathrm{~h}$. The culture supernatants were collected and subjected to cytokine array (R\&D Systems, Minneapolis, MN, USA) according to the manufacturer's instruction. Briefly, the culture supernatants were centrifuged at 10,000 $\times \mathrm{g}$ for $2 \mathrm{~min}$ and the supernatants were transferred to new tubes. Human cytokine array panel A membranes (R\&D Systems) were incubated with FW-treated (Sup+) or -untreated (Sup-) HSC3 cell culture supernatants. After washing, the membranes were further incubated with a detection Ab cocktail for $1 \mathrm{~h}$. Spot detection was performed with streptavidinhorseradish peroxidase incubation, followed by the use of the Enhanced Chemiluminescent (ECL) kit (GE Healthcare, Tokyo, Japan). The intensity of the spot was measured by the NIH Image software. The Sup+ and Sup- were subjected to IL-1a concentration measurements using the DuoSet enzyme-linked immunosorbent assay (ELISA) development system (R\&D Systems). The absorbance was measured on a microplate reader (model 3550; Bio-Rad, Tokyo, Japan).

\section{Immunoprecipitation and Western blotting}

The HSC3 cells were lysed with cell lysis buffer (50 mM Tris- $\mathrm{HCl}$, pH 7.5, $150 \mathrm{mM} \mathrm{NaCl}$, and 0.5\% 
TritonX-100). The HSC3 cell lysate, Sup+, and Sup were subjected to immunoprecipitation (IP) using rabbit anti-human IL-1a Ab (Abcam, Cambridge, UK) for $18 \mathrm{~h}$ at $4{ }^{\circ} \mathrm{C}$. Ten $\mu \mathrm{l}$ of protein G-sepharose was added to the samples, which were rotated (Yamato Scientific, Tokyo, Japan) $50 \mathrm{rpm}$ for $2 \mathrm{~h}$. After rotation, the samples were washed with PBS or cell lysis buffer and loaded on to a $15 \%$ SDS-PAGE gel. The separated samples were transferred to a membrane and subjected to Western blotting (WB), which was performed using rabbit anti-human mIL-1a $\mathrm{Ab}$ (Abcam) followed by horseradish peroxidaseconjugated goat anti-rabbit immunoglobulin (Ig)G $(\mathrm{H}+\mathrm{L}) \mathrm{Ab}$. The bands were detected using an ECL kit (GE Healthcare).

\section{Immunofluorescence staining}

The HSC 3 cells were plated on to coverslips at a density of $5 \times 10^{4} / 24$ well/coverslip. After three washes with PBS, they were treated with or without FW for $30 \mathrm{~s}$. The cells were further incubated with $10 \%$ FCS-RPMI for $3 \mathrm{~h}$ following which, they were fixed with $4 \%$ paraformaldehyde for $15 \mathrm{~min}$. After washing with PBS, the permeabilization of the cells was performed by incubating them with $1 \%$ Triton X-100 solution (1\% Triton X-100 in PBS) for $30 \mathrm{~min}$. The cells were incubated with $1 \%$ BSA-PBS for $1 \mathrm{~h}$ to block the non-specific reaction; subsequently, they were incubated with rabbit anti-human IL-1a Ab (Abcam; $\times 100$ dilution with $1 \%$ BSA-PBS) for another $18 \mathrm{~h}$. After three wash with PBS, the cells were incubated with FITC-conjugated goat anti-rabbit IgG for $18 \mathrm{~h}$. The cells were extensively washed with PBS and mounted on glass slides using Fluoroshield with DAPI (Gene Tex, California, USA). Images were taken using a fluorescence microscope (All-in-one Fluorescence Microscope, Keyence, Osaka, Japan).

\section{Statistical analysis}

Statistically significant differences were determined by two-tailed Student's t-tests and Tukey's test. A p-value $<0.05$ was considered significant. All data are plotted as means \pm standard deviation (SD).

\section{Results}

\section{Augmentation of IL- 1 a secretion following FW treatment}

A cytokine array experiment was conducted to examine the FW-mediated release of cytokines. The HSC 3 cells were treated with or without FW and cultured for $18 \mathrm{~h}$ following which, the culture supernatants were collected and incubated using a factor-spotted membrane. Among the cytokines, IL-1a secretion was significantly augmented in the
FW-treated culture supernatant; a 1.5-2-fold increase in the intensity of IL-1a spotting was observed in Sup+ (Fig. 1a, upper panel) when compared to that in Sup- (Fig. 1a, lower panel). To confirm the enhanced secretion of IL-1a, Sup + and Sup - were collected after 30, 60, and $180 \mathrm{~min}$ of $\mathrm{FW}$ treatment and subjected to ELISA. In Sup -, a gradual increase in the concentration of IL-1a from $77.5 \pm 8.6 \mathrm{pg} / \mathrm{mL}$ at 30 $\mathrm{min}$ to $111.2 \pm 54.5 \mathrm{pg} / \mathrm{mL}$ at $180 \mathrm{~min}$ was noted (Fig. $1 b)$. In contrast, FW treatment markedly enhanced IL-1a secretion in Sup + from $375.0 \pm 148.3 \mathrm{pg} / \mathrm{mL}$ at $30 \mathrm{~min}$ to $601.3 \pm 137.4 \mathrm{pg} / \mathrm{mL}$ at $60 \mathrm{~min}$ and $859.0 \pm$ $352.8 \mathrm{pg} / \mathrm{mL}$ at $180 \mathrm{~min}$ (Fig. 1b).

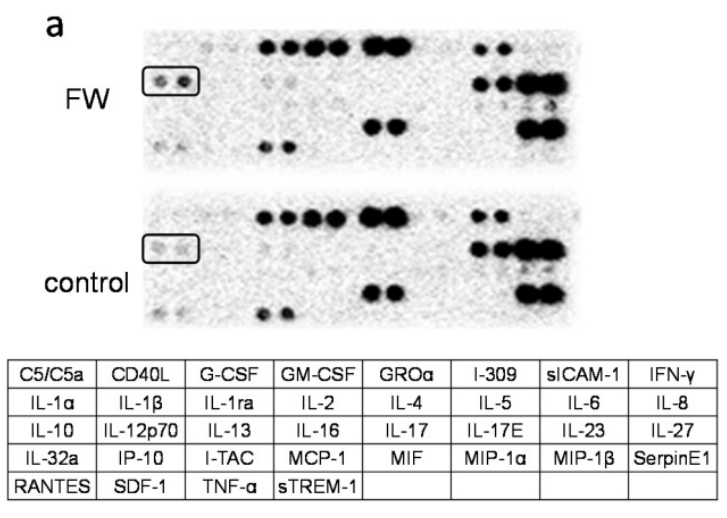

b

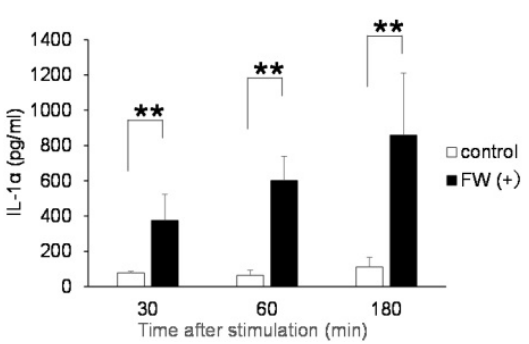

Figure 1. IL-1 $\alpha$ secretion by the HSC3 cells. a. Cytokine array showing the amount of IL-1 $\alpha$ secreted in cells treated with (upper panel) or without FW (lower panel) for $30 \mathrm{~s}$. The IL-1 $\alpha$ spots are indicated by the rectangles. The table below shows the spot position for each cytokine in the array. b. Bar graph showing enhanced secretion of IL- $1 \alpha$ in the supernatants obtained from HSC3 cells treated with (black bars) or without FW (clear bars) for 30,60 , and 180 min via ELISA. The mean \pm SD of at least five independent experiments are shown. ${ }^{* *}, p<0.05$.

\section{New protein synthesis was not involved in the augmented secretion of IL-1 $\alpha$}

IL-1a is a typical alarmin, and the increased IL-1a in Sup + following FW treatment is thought to have been derived from intracellular stores. Consequently, the inhibition of new protein synthesis by $\mathrm{CHX}$ treatment might not affect the amount of IL-1a released in Sup +. To test this possibility, IL-1a concentration was measured after pre-incubation with or without CHX; pre-incubation with $1 \mu \mathrm{M}(109 \pm 29$ $\mathrm{pg} / \mathrm{ml})$ or $10 \mu \mathrm{M}(119 \pm 30 \mathrm{pg} / \mathrm{ml})$ of $\mathrm{CHX}$ did not affect the spontaneous secretion of IL-1a when 
compared with the CHX non-treated cells $(0 \mu \mathrm{M}: 124 \pm$ $55 \mathrm{pg} / \mathrm{ml})$. FW stimulation after $\mathrm{CHX}$ pre-treatment augmented IL-1a secretion to levels (CHX 1 $\mu \mathrm{M}$ : $273 \pm$ $21 \mathrm{pg} / \mathrm{ml}, 10 \mu \mathrm{M}: 284 \pm 70 \mathrm{pg} / \mathrm{ml}$ ) equivalent to that in the CHX non-treated FW stimulated controls (347 \pm $27 \mathrm{pg} / \mathrm{ml}$ ) (Fig. 2a). The IL-1a secretion was further examined after 30, 60, and 180 min of FW stimulation with or without CHX pre-treatment; although a reduction in IL-1a secretion was observed after 30 min of FW stimulation following $\mathrm{CHX}$ pre-treatment, the levels were, subsequently, found to increase in a time-dependent manner (Fig. 2b). At $180 \mathrm{~min}$, the amount of IL-1a secreted was similar to those in the CHX non-treated and FW-stimulated samples (Fig. $2 b)$. These results indicated that the augmented IL-1a might be derived from intracellular storage sites. To confirm this assumption, the amount of IL-1a in the cell lysates was measured. The IL-1a concentration in cells without FW stimulation was $694.7 \pm 139.2 \mathrm{pg} / \mathrm{ml}$ (Fig. 3a), whereas a drastic reduction to $111.6 \pm 80.3$ $\mathrm{pg} / \mathrm{ml}$ was observed in cells treated with FW for $18 \mathrm{~h}$ (Fig. 3a). Furthermore, pre-treatment of HSC3 with or without anti-IL-1R1 Ab prior to FW stimulation did not affect the amount of IL-1a secreted from the cells (Fig. 3b), thus eliminating the possible contribution of autocrine IL-1a augmentation.

a

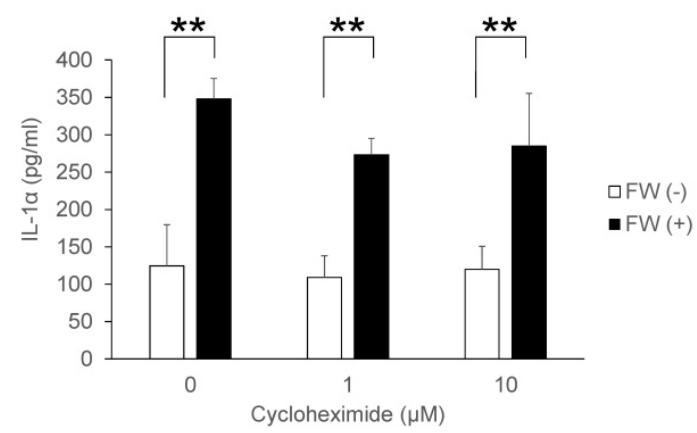

b

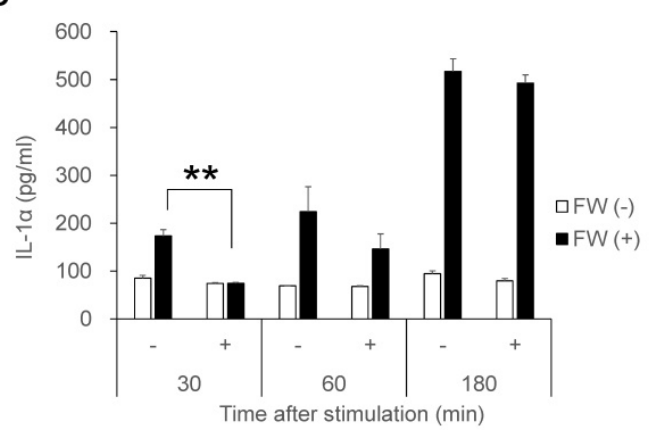

Figure 2. IL-1 $\alpha$ secretion in cells treated with $\mathrm{CHX}$. a. Bar graphs showing equivalent amounts of IL-1 $\alpha$ secretion in the cells treated with 1 and $10 \mu \mathrm{M} \mathrm{CHX}$ when compared with the non-treated controls. b. Bar graph showing a time-dependent increase in IL-1 $\alpha$ in the supernatants of $\mathrm{HSC} 3$ cells pre-treated with or without $1 \mu \mathrm{M} \mathrm{CHX}$ and stimulated with $\mathrm{FW}$ for $30 \mathrm{~s}$. The culture supernatants were collected after 30,60 , and 180 min and subjected to IL-1 $\alpha$ ELISA. The mean \pm $\mathrm{SD}$ of at least three independent experiments are shown. **, $p<0.05$. a

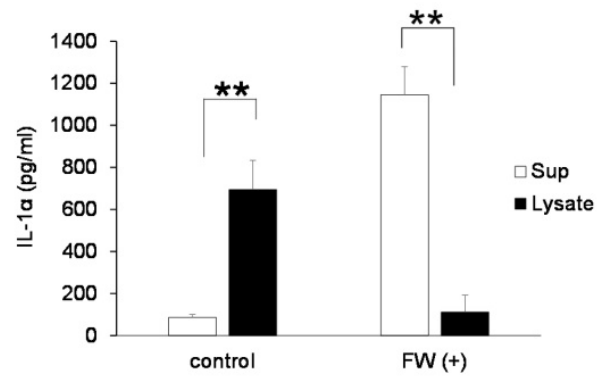

b

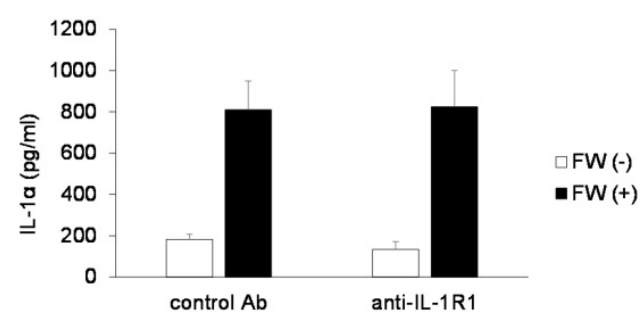

Figure 3. IL-1 $\alpha$ derived from intracellular storages. a. Bar graph showing IL-1 la levels in the cell lysates and supernatants of the HSC3 cells that were stimulated with (FW (+)) or without FW (control) for $30 \mathrm{~s}$, and cultured for a further $18 \mathrm{~h}$. The culture supernatants (Sup) and cell lysates (lysate) were collected and subjected to ELISA. b. HSC3 cells were pre-incubated with anti-IL-IRI Ab or control Ab for $2 \mathrm{~h}$ and treated with FW for 30 s. The Sup was collected and subjected to ELISA. The mean $\pm S D$ of at least three independent experiments are shown. ${ }^{* *}, p<0.05$.

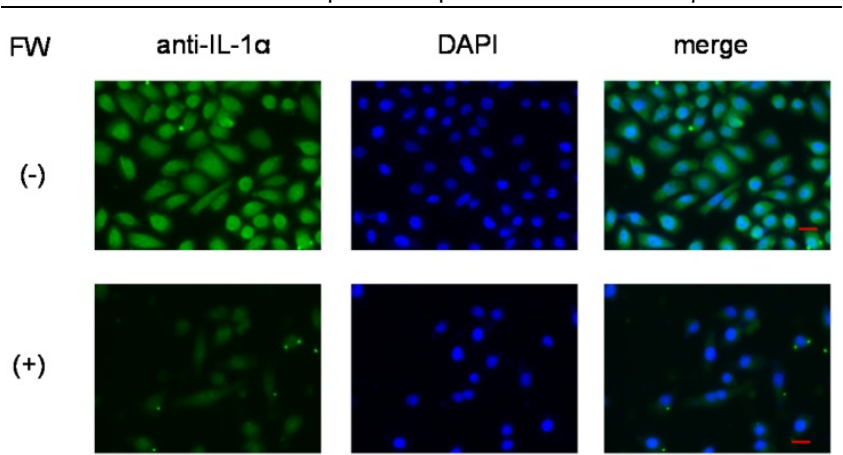

Figure 4. Immunofluorescence staining of $\mathrm{HSC} 3$ cells. After treatment with (lower panel, (+)) or without FW (upper panel, (-)), the cells were cultured for $3 \mathrm{~h}$. Those without FW treatment showed strong fluorescence (-), mainly in the nucleus, and to a lesser extent in the cytoplasm. No fluorescence was observed in the FW-treated cells $(+)$. The representative data of three independent experiments are shown.

\section{Secretion of IL- 1 a from intracellular stores}

To further confirm that IL-1a was released from intracellular stores, the HSC 3 cells were subjected to immunofluorescence staining. Those without FW treatment showed strong fluorescence (Fig. 4, upper panel), mainly in the nucleus, and to a lesser extent in the cytoplasm. On the other hand, no fluorescence was observed in the FW-treated cells (Fig. 4, lower panel).

\section{IL-1 $\alpha$ forms released in response to FW}

To further examine the IL-1a molecular form secreted in response to FW, we performed WB. As shown in Figure 5a, only a $33 \mathrm{kDa}$ band was detected in the HSC3 cell lysate. When Sup- was subjected to 
IP-WB, only a single $33 \mathrm{kDa}$ band was detected (Fig. $5 \mathrm{~b}$, left panel, (-)). On the other hand, two closely-loaded bands approximately $19 \mathrm{kDa}$ in size were observed, in addition to the $33 \mathrm{kDa}$ band, in FW-stimulated Sup+ (Fig. 5b, right panel, $(+)$ ). These results confirmed that the HSC3 cells secreted not only pIL-1a but also the $19 \mathrm{kDa}$ form of IL-1a in response to FW.

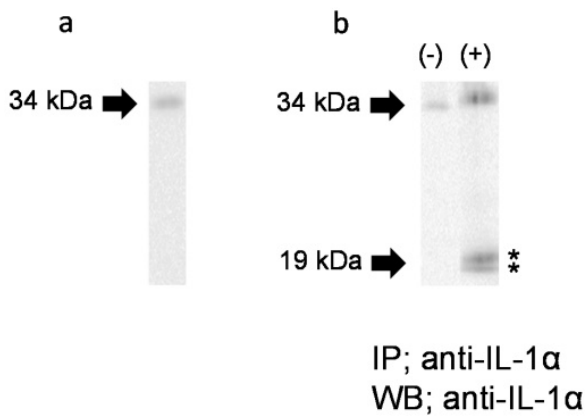

Figure 5. Molecular forms of IL-1 $\alpha$ secreted in response to FW. a. A $33 \mathrm{kDa}$ band was detected in the $\mathrm{HSC} 3$ cell lysate. $\mathrm{b}$. The $33 \mathrm{kDa}$ band was detected in $\mathrm{FW}$ non-stimulated HSC3 supernatant (left panel, (-)). In addition to the $33 \mathrm{kDa}$ band, two bands $(*)$ approximately $19 \mathrm{kDa}$ in size were detected in $\mathrm{FW}$ stimulated supernatant $(+)$. The representative of five independent experiments are shown.

a

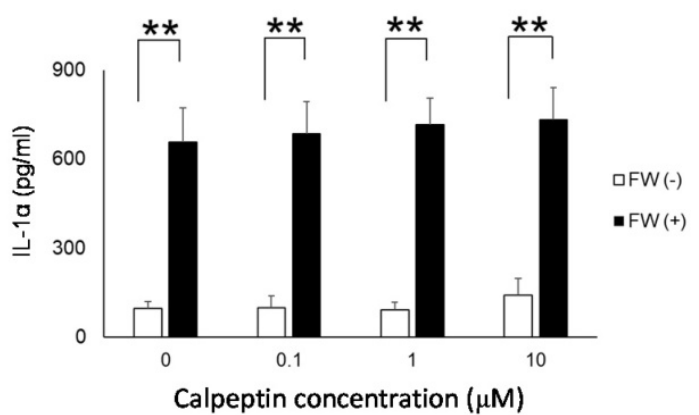

b

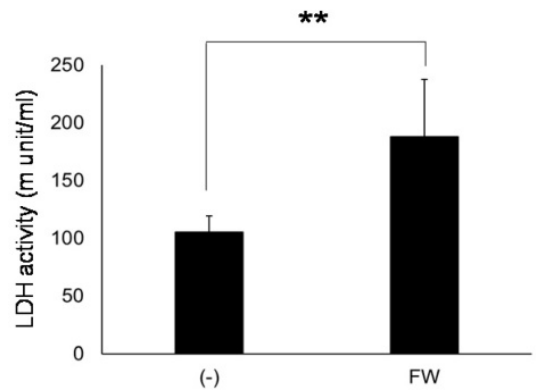

Figure 6. a. Calpeptin pre-treatment did not affect the amount of IL-1 $\alpha$ released into the supernatants. $\mathrm{HSC} 3$ was pre-incubated with $0,0.1,1$ and 10 $\mu \mathrm{M}$ of calpeptin for $1 \mathrm{~h}$ and further treated with FW for $30 \mathrm{sec}$. The supernatants were collected after $3 \mathrm{~h}$ of incubation and IL-1 $\alpha$ concentration was measured with ELISA. Calpeptin pre-treatment did not affect IL-l $\alpha$ concentration in the culture supernatants. b. The extent of call damage. $\mathrm{HSC} 3$ was treated with or without $\mathrm{FW}$ for $30 \mathrm{sec}$ and further cultured for $3 \mathrm{~h}$. The LDH activity in the culture supernatants was measured with LDH activity assay kit. The LDH activity was augmented after $3 \mathrm{~h}$ of culture following the FW treatment. The mean \pm SD of at least three independent experiments are shown. **, $p<0.05$

\section{Calpain inhibition and lactate dehydrogenase release}

To further examine the contribution of pIL-1a processing enzyme calpain to IL-1a release from HSC3, the cells were pre-treated with or without calpeptin, a specific calpain inhibitor. As shown in Fig. 6a, IL-1a released in response to FW-stimulation was not affected by the calpeptin pre-treatment. Furthermore, the extent of cell damage after FW-treatment was examined by measuring the amount of LDH released in the culture supernatants. After $3 \mathrm{~h}$ of FW-treatment, the LDH released into the culture supernatants increased significantly (FWtreated; $188.2 \pm 49.4$ vs control; $105.6 \pm 13.8$ ) (Fig. 6b).

\section{Discussion}

IL-1a and IL-1 $\beta$ share common molecular and functional properties as members of the IL- 1 superfamily [17]. Although they have only $27 \%$ homology at the amino acid level, they can bind to the same receptor (IL-1R1) due to similarities in the three-dimensional structure [18]. They are initially synthesized as precursors (pIL-1 $\alpha$ and pIL-1 $\beta$ ) that enzymatically cleave to produce the mature forms (mIL-1 $\alpha$ and mIL-1 $\beta$ ) within the cell. Moreover, both molecules do not have a signal sequence and are secreted outside the cell via the unconventional ER-Golgi-independent pathway [10]. Despite these similarities, FW treatment only induced the secretion of IL-1a (Fig. 1a), whereas the secretion of the other 34 cytokines was not augmented by FW.

FW-mediated IL-1a secretion was increased time-dependently (Fig. 1b). To exclude the possible contribution of autocrine IL-1a augmentation, the HSC3 cells were treated with anti-IL-1R1 Ab. As shown in Fig. 3b, the Ab did not affect the amount of IL-1a released by FW. Although FW-induced IL-1a secretion was slightly inhibited at the $30 \mathrm{~min}$ time point following $\mathrm{CHX}$ pre-treatment, it increased to levels similar to those seen in the CHX non-treated controls after $180 \mathrm{~min}$ of culture (Fig. 2b). Taken together, these results showed that the augmented IL-1a in Sup+ is derived from intracellular storage sites. Immunofluorescence staining revealed nuclear, and to a lesser extent, cytoplasmic localization of IL-1a in the HSC3 cells (Fig. 4). Due to the existence of NLS in the N-terminal region, pIL-1a preferentially localizes in the nucleus [19]. In addition, pIL-1a has been shown to localize on the cell membrane through a lectin-like interactions [20] or myristoylation [21]. More importantly, nuclear localization and release of IL-1 $\alpha$ is affected by the acetylation of NLS [22, 23]. FW treatment drastically reduced the fluorescence intensity in all these compartments (Fig. 4) and the mechanisms underlying should be examined by further experiments.

IL-1a is detected in a variety of non-myeloid cells such as keratinocytes, endothelial cells, and 
fibroblasts [24]. The enzymes that cleave pIL-1a into ppIL-1 $\alpha$ and mIL-1 $\alpha$ are calpain $[25,26]$, granzyme B (GzmB), elastase, mast cell chimase and thrombin [27, $28,29]$. Due to the abundance of calpain inhibitors, non-myeloid cells cannot produce mIL-1a inside the cells [26]. Consistent with these reports, only the 33 $\mathrm{kDa}$ pIL-1a was detected in the HSC3 cells in this study (Fig. 5a).

The IP-WB experiment was performed to reveal the form of IL-1a released in response to FW. Surprisingly, two bands of about $19 \mathrm{kDa}$ were detected in Sup+, in addition to the $33 \mathrm{kDa}$ band (Fig. $5 b)$. Theoretically, the $A b$ used in IP can recognize pIL-1a, ppIL-1a, and mIL-1a because it is obtained by immunizing pIL-1a. Hence, the two additional bands detected in Sup + might correspond to mIL-1a and ppIL-1a. Previously, we developed a polyclonal Ab that recognizes ppIL-1a by immunizing rats with histidine-tagged ppIL-1a [30]. However, both bands could not be recognized by this Ab (data not shown) due to its low sensitivity. Nonetheless, additional studies are required to determine the nature of these two bands.

Both pIL-1 $\alpha$ and mIL-1 $\alpha$ are biologically active in the extracellular milieu and can bind to IL-1R1 [15]. Furthermore, as shown in this study, pIL-1a was secreted by HSC3 in response to FW. These facts suggested that the secreted pIL-1a could interact with its cleaving enzyme, such as GzmB. GzmB is secreted into the extracellular space, and its level in the serum is significantly increased in patients with rheumatoid arthritis [31]. Thus, the cleavage of pIL-1a could result in the generation of $\mathrm{pp}$ and mIL-1a, extracellularly. Although the extracellular function of ppIL-1a has never been examined, this phenomenon could increase the immunological role of IL-1a. The mechanisms involved in the release of $19 \mathrm{kDa}$ IL-1a in the Sup+ are unknown. The treatment of macrophages with calcium ionophore has been shown to activate calpain, a $\mathrm{Ca}^{2+}$-dependent enzyme [32], resulting in the cleavage of pIL-1a [33]. FW might induce the influx or release of calcium from the intracellular storage sites, such as the mitochondria, and generate pp and mIL-1a. To further examine the contribution of calpain on $19 \mathrm{kDa}$ IL-1a release, HSC3 was pre-incubated with calpain inhibitor calpeptin prior to FW stimulation. However, calpeptin did not affect the amount of IL-1a released in response to FW treatment (Fig. 6a). As IL-1a is released not only from dying cells (8. 9) but also from live cells [22, 23, 34, 35, $36,37]$, we further attempted to examine the extent of cell damage by measuring LDH activity. As the LDH activity was significantly augmented in FW-treated cells (Fig. 6b), the cells are expected to be damaged to some extent.
FW treatment augmented the release of IL-1a from HSC3. The augmentation was not accompanied with increased IL-1a mRNA transcription. Anti-IL1R1 Ab blocking did not affect IL-1a. Moreover, inhibition of new protein synthesis by $\mathrm{CHX}$ had no effects on IL-1a release. Taken all these results together, we speculated here that IL-1a released in response to FW treatment is passively released by the damaged cells. Recently, IL-1a was demonstrated to detect DNA damage in living cells and actively released to the outside of the cells to evoke the inflammatory reactions [38]. With these functions, IL-1a is denoted as "Stressorin". FW-treatment totally abolished the cellular IL-1a by immunofluorescence staining (Fig. 4) and we could not trace the path of IL-1a movement inside the cells. For these reasons, we could not detect the stressorin function of IL-1a in the present study. However, the properties and mechanisms involved in the generation of the two 19 $\mathrm{kDa}$ bands should be examined in future experiments.

\section{Conclusion}

In the present study, FW treatment was shown to induce IL-1a secretion in HSC3 cells. The secreted form contained $33 \mathrm{kDa}$ precursor form and two 19 $\mathrm{kDa}$ forms; hence, FW might be able to enhance the cleavage of pIL-1a. Elucidation of the mechanisms underlying this phenomenon should extend our understanding of the properties of IL-1a and enlighten us about the benefits of using FW.

\section{Acknowledgements}

This work was supported by a Grant-in-aid for Scientific Research (C) (2020-2022) and a Grant-in-aid for Young Scientists (2019-2021). The Research grant from Scholarship Fund for Young/Women Researchers; a grant from the Dental Research Center, Nihon University School of Dentistry. The authors do not have any conflict of interest.

\section{Competing Interests}

The authors have declared that no competing interest exists.

\section{References}

1. Ito K, Nishida T, Murai S. Inhibitory effects of acid water prepared by an electrolysis apparatus on early plaque formation on specimens of dentine. J Clin Periodontol. 1996; 23: 471-6.

2. Shimada K, Ito K, Murai S. A comparison of the bactericidal effects and cytotoxic activity of three types of oxidizing water, prepared by electrolysis, as chemical dental plaque control agents. Int J Antimicrob Agents. 2000; 15: 49-53.

3. Okamura T, Tamura M, Suguro H, et al. Bactericidal and cytotoxic effects of acid-electrolyzed functional water. J Oral Sci. 2019; 61: 512-5.

4. Nakae $H$, Inaba $H$. Electrolyzed strong acid aqueous solution irrigation promotes wound healing in a burn wound model. Artif Organs. 2000; 24:544-6.

5. Motozawa K, Motoyoshi M, Saiki A, et al. Functional comparison of high and low molecular weight basic fibroblast growth factors. J Cell Biochem. 2018; 119: 7818-26. 
6. Saiki A, Motoyoshi M, Motozawa K, et al. EMMPRIN Inhibits bFGF-Induced IL-6 Secretion in an Osteoblastic Cell Line, MC3T3-E1. Int J Med Sci. 2017; 4: 1173-80.

7. Kusunoki M, Sata E, Nishio $\mathrm{K}$, et al. Acid-electrolyzed functional water induces extracellular matrix metalloproteinase inducer, a possible novel alarmin, secretion from oral squamous cell carcinoma cell lines. Int J Med Sci. 2018; 15: 1365-72.

8. Oppenheim JJ, Yang D. Alarmins: chemotactic activators of immune responses. Curr Opin Immunol. 2005; 17: 359-65.

9. Di Paolo NC, Shayakhmetov DM. Interleukin 1a and the inflammatory process. Nat Immunol. 2016; 17: 906-13.

10. Monteleone M, Stow JL, Schroder K. Mechanisms of unconventional secretion of IL-1 family cytokines. Cytokine. 2015; 74: 213-8.

11. Werman A, Werman-Venkert $R$, White $R$, et al. The precursor form of IL-1 alpha is an intracrine proinflammatory activator of transcription. Proc Natl Acad Sci USA. 2004; 101: 2434-9.

12. Rider P, Carmi $Y$, Guttman O, et al. IL-1 $\alpha$ and IL-1 $\beta$ recruit different myeloid cells and promote different stages of sterile inflammation. J Immunol. 2011; 187: $4835-43$

13. Cohen I, Rider P, Carmi Y, et al. Differential release of chromatin-bound IL-1alpha discriminates between necrotic and apoptotic cell death by the ability to induce sterile inflammation. Proc Natl Acad Sci USA. 2010; 107: 2574-9.

14. England H, Summersgill HR, Edye ME, et al. Release of interleukin-1a or interleukin-1 $\beta$ depends on mechanism of cell death. J Biol Chem. 2014; 289: 15942-50.

15. Kim B, Lee Y, Kim E, et al. The interleukin-1a precursor is biologically active and is likely a key alarmin in the IL-1 family of cytokines. Front Immunol. 2013; 4: 391.

16. Chen CJ, Kono H, Golenbock D, et al. Identification of a key pathway required for the sterile inflammatory response triggered by dying cells. Nat Med. 2007; 13. 851-6.

17. Dinarello CA. Immunological and inflammatory functions of the interleukin-1 family. Annu Rev Immunol. 2009; 27: 519-50.

18. Dinarello CA. Biologic basis for interleukin-1 in disease. Blood. 1996; 87: 2095147.

19. Lamacchia C, Rodriguez E, Palmer G, et al. Endogenous IL-1a is a chromatin-associated protein in mouse macrophages. Cytokine. 2013; 63: $135-44$.

20. Brody DT, Durum SK. Membrane IL-1: IL-1 alpha precursor binds to the plasma membrane via a lectin-like interaction. J Immunol. 1989; 143: 1183-7.

21. Stevenson FT, Bursten SL, Fanton C, et al. The 31-kDa precursor of interleukin 1 alpha is myristoylated on specific lysines within the $16-\mathrm{kDa} \mathrm{N}$-terminal propiece. Proc Natl Acad Sci U S A. 1993; 90: 7245-9.

22. Cohen I, Idan C, Rider P, et al. IL-1a is a DNA damage sensor linking genotoxic stress signaling to sterile inflammation and innate immunity. Sci Rep. 2015; 5: 14756.

23. Cohen I, Rider P, Vornov E, et al. Corrigendum: IL-1a is a DNA damage sensor linking genotoxic stress signaling to sterile inflammation and innate immunity. Sci Rep. 2016; 6: 19100

24. Bertheloot D, Latz E. HMGB1, IL-1a, IL-33 and S100 Proteins: Dual-Function Alarmins. Cell Mol Immunol. 2017; 14: 43-64.

25. Kobayashi Y, Yamamoto K, Saido T, et al. Identification of calcium-activated neutral protease as a processing enzyme of human interleukin 1 alpha. Proc Natl Acad Sci USA. 1990; 87: 5548-52.

26. Carruth LM, Demczuk S, Mizels SB. Involvement of a calpain-like protease in the processing of the murine interleukin la precursor. J Biol Chem. 1991; 266: $12162-7$

27. Afonina IS, Tynan GA, Logue SE, et al. Granzyme B-dependent proteolysis acts as a switch to enhance the proinflammatory activity of IL-1a. Mol Cell. 2011; 44: 265-78.

28. Afonina IS, Muller C, Martin SJ et al. Proteolytic processing of interleukin-1 family cytokines: Variations on a common theme. Immunity. 2015; 42: 991-1004.

29. Burzynski LC, Humphry M, Pyrillou K, et al. The coagulation and immune systems are directly linked through the activation of interleukin-1a by thrombin. Immunity. 2019; 50: 1033-42.

30. Sata E, Takada L, Kaetsu R, et al. A new enzyme-linked immunosorbent assay system against the N-terminal propiece of interleukin-1a. J Oral Sci. 2020; 62: 340-3.

31. Spaeny-Dekking EH, Hanna WL, Wolbink AM, et al. Extracellular granzymes $A$ and $B$ in humans: detection of native species during CTL responses in vitro and in vivo. J Immunol. 1998; 160: 3610-6.

32. Goll DE, Thompson VF, Li H, et al. The calpain system. Physiol Rev. 2003; 83: 731-801.

33. Suttles J, Giri JG, Mizel SB. Enhancement of IL-1 Secretion and Processing by Calcium Ionophores. J Immunol. 1990; 144: 175-82.

34. Feldmeyer L, Keller M, Niklaus G, et al. The Inflammasome mediates UVB-induced activation and secretion of interleukin-1 $\beta$ by keratinocytes. Curr Biol. 2007; 17: 1140-5.

35. Orjalo AV, Bhaumik D, Gengler BK, et al. Cell surface-bound IL-1 is an upstream regulator of the senescence-associated IL-6/IL-8 cytokine network. Proc Natl Acad Sci USA. 2009; 106: 17031-6.
36. Freigang S, Ampenberger F, Weiss A, et al. Fatty acid-induced mitochondrial uncoupling elicits inflammasome- independent IL-1a and sterile vascular inflammation in atherosclerosis. Nat Immunol. 2013; 14: 1045-53.

37. Salti $T$, Khazim $K$, Haddad $R$, et al. Glucose induces IL-1a-dependent inflammation and extracellular matrix proteins expression and deposition in renal tubular epithelial cells in diabetic kidney disease. Front Immunol. 2020; 11: 1270.

38. Rider P, Voronov E, Dinarello CA, et al. Alarmins: Feel the stress. J Immunol. 2017; 198: 1395-402. 